\title{
Damage Diagnosis of Steel Truss Bridges under Varying Environmental And Loading Conditions
}

\author{
Kundan Kumar and Prabir Kumar Biswas \\ Department of Electronics and Electrical Communication Engineering, Indian Institute of Technology Kharagpur, \\ Kharagpur-721302, India. \\ Nirjhar Dhang \\ Department of Civil Engineering, Indian Institute of Technology Kharagpur, Kharagpur-721302, India.
}

\begin{abstract}
(Received 27 June 2016; accepted 31 January 2017)
In this paper, we propose a damage detection and localization algorithm for steel truss bridges using a data-driven approach under varying environmental and loading conditions. A typical steel truss bridge is simulated in ANSYS for data generation. Damage is introduced by reducing the stiffness of one or more members of the truss bridge. The simulated acceleration time-history signals are used for the purpose of damage diagnosis purpose. Vibration data collected from healthy bridges are processed through principal component analysis (PCA) to find the reduced size weighted feature vectors in model space. Unknown test vibration data (healthy or damaged) finds the closest match of its reduced size model from the training database containing only healthy vibration data. The residual error between the spread of closest healthy vibration data and unknown test vibration data is processed to determine damage location and severity of the damage to the structure. A comparative study between a proper orthogonal decomposition (POD) based damage detection algorithm and proposed algorithm is presented. The results show that the proposed algorithm is efficient to identify the damage location and assess the severity of damage, called as the Damage Index (DI), under varying environmental and moving load conditions.
\end{abstract}

\section{INTRODUCTION}

Many infrastructures that have reached or are the end of approaching their design life are still under excessive repetitive use. These structures require continuous monitoring over their remaining service life. In general, the physical properties of the structure like mass, stiffness, damping coefficient, etc. change over time due to various factors e.g. aging effect, cracks, excessive load and other types of damage. This results in changes in modal properties and other structural behaviour. ${ }^{1}$ The vibration responses of the structure capture the effect of the changes in these properties. Bridge health monitoring attracted the researchers to the development of a vibration based health monitoring system to identify the damage on the bridge at the earliest possible stage using its dynamic behaviour analysis. ${ }^{2-5}$ Doebling et al. have reviewed several strategies for vibration-based damage detection for bridges. ${ }^{6} \mathrm{~A}$ detailed study on different approaches implemented for Structure Health Monitoring (SHM) can be found in the literature. ${ }^{7,8}$ In SHM, many researchers have addressed the damage detection problem as outlier detection/novelty detection in varying operational and environmental conditions. ${ }^{9-13}$ Meruane and Heylen proposed a model-based damage detection approach to detect, locate and quantify the damage on a three span bridge under varying temperatures. ${ }^{14}$ In these methods, operational and environmental variabilities are considered as implicitly embedded in structural responses. Kim et al. have investigated the feasibility of traffic-induced vibration signals for damage detection in real steel truss bridges. ${ }^{12}$ Recently, train-induced vibrations of a simplified railway bridge model were used by Shu et al. to detect damage based on statistical properties of structural dynamic responses such as variances and covariances. ${ }^{15}$ However, operational and environmental variabilities are not considered, and they should be considered. Yarnold and Moon have established a relationship between temperature change and the resulting strains and displacement of the structure. ${ }^{16}$ They have evaluated their approach using long-term monitoring data from a long-span steel tied arch bridge. Li and Hao have successfully detected joint damage in a laboratory scaled steel truss bridge based on the time-frequency analysis of free vibration data. ${ }^{17}$ There is very limited work on damage diagnosis of steel truss bridges using traffic-induced vibration data under varying environmental conditions. In this paper, a damage detection approach is presented for steel truss bridges using the moving load response of the structure in varying environmental temperature and loading conditions.

In the last few decades, many bridge health monitoring systems have been implemented based on vibration signal analysis wherein the vibration signals are collected from more than 100 sensors placed on the bridge. ${ }^{18}$ These sensors produce a significant amount of vibration data. The analysis of all the vibration data together is a very crucial task. According to Cavadas et $a l$., a data-driven approach is suitable to handle all these vibration signals simultaneously. ${ }^{19}$ The data-driven approach tracks the change in signal only; hence, it is well suited to capture the relevant information for continuous monitoring of bridge structures. They have applied a data-driven approach to the problem of detecting damage under varying load conditions and moving load measurements. They have used moving principal component analysis (MPCA) to find the discrimination between eigenvectors of the covariance matrices of the data corresponding to the undamaged and damaged structure for damage detection and severity measurement. However, they have not considered the change in vibration characteristics due to varying environmental conditions. 
Lanta and Grosso used proper orthogonal decomposition (POD) to find the damage in a bridge structure. ${ }^{20}$ The PODbased techniques include principal component analysis (PCA) and singular value decomposition (SVD). POD reduces the dimensionality of the data samples, such that most of the energy contents of the data are preserved in reduced size data. It has been shown that POD-based techniques, like SVD, are limited to producing a good approximation of mode shape of the structure for a free or lightly damped vibration. ${ }^{21}$ Rao et al. combined POD based technique with self-adaptive differential evolution algorithm to detect damage considering environmental variabilities. ${ }^{22}$ Shane and Jha have applied SVD to localize the fault in a cantilever plate with an impact load. ${ }^{23}$ Brunell and Kim have observed that the global response of a truss bridge was significantly influenced by the local damage of the truss members. ${ }^{24}$ However, the extent of such influence depends on the position of the damaged member in the truss bridge. For example, for the same extent of damage, a damaged diagonal member will have a different effect on the global response than a damaged vertical member. Truss bridges have a lack of redundancy that leads to premature failure. ${ }^{25}$ These structures are statically determinate because support reactions and member forces can be calculated using only the equation of static equilibrium. The failure of a single truss member usually increases the burden on other truss members leading to failure of multiple truss members and ultimately the failure of the entire structure. Therefore, continuous monitoring of the truss bridge structure is required to detect the damage at the earliest possible stage.

This paper focuses on continuous monitoring of steel truss bridge structures through the analysis of vibration signals. Acceleration time-history signals as vibration data are collected from the simulated healthy bridge under various environmental temperature and loading conditions for the purposes of modelling. The vibration data are collected from multiple sensors placed at various web joints on the steel truss bridge. A damage detection and localization algorithm is presented based on the data-driven approach using PCA. PCA is used in a very efficient way to extract the reduced size weighted feature vectors that model the dynamic behaviour of the structure under various environmental and loading conditions. We call this model the model space. For the damage diagnosis, the test vibration data is represented in the same reduced model space, and the model data closest to the test vibration data is selected in the model space. This step is necessary to ensure that the environmental and loading condition of the test vibration data and the model data are similar. The residual standard error between the spread of the test vibration data and corresponding healthy model data in the original data space determines the discrepancy, due to the change in structure behaviour. The closest model data is used to normalize the residual standard error to obtain the local damage index (LDI) at each sensor location. The damage index (DI) is computed by accumulating all LDIs to estimate the damage severity of the whole structure. Three levels of damage are investigated for several damage locations on the structure. The main objective of the proposed method is to detect and localize the damage on the truss structures in a computationally efficient way with a fewer number of sensors under varying environmental temperature and loading conditions.

The paper is organized as follows. The proposed damage di- agnosis approach is discussed in the Section 2. In Section 3.1, a numerical model of a typical steel truss bridge used for case study is discussed. The collection of time-history responses of the structure in different environmental and loading conditions are presented in Section 3.2. The application of the damage detection algorithm to the truss bridge model and a comparative analysis with other reported algorithm is presented in the results and discussion of Section 4. Finally, the work is concluded in Section 5.

\section{PROPOSED DAMAGE DIAGNOSIS APPROACH}

A continuous health monitoring technique of truss bridge structures employing a number of sensors placed at web joints is presented here. The sensors generate a huge amount of vibration data when the bridge is subjected to a moving load. A data-driven approach is adopted to process the huge amount of vibration data.

Data representation of the multiple sensors data in a matrix facilitates decoding the sensor location with respect to the column number of the matrix. Consider the data sample, $A \in \Re^{n \times N}$, that is expressed as:

$$
A=\left[\begin{array}{cccc}
x_{11} & x_{12} & \cdots & x_{1 N} \\
x_{21} & x_{22} & \cdots & x_{2 N} \\
\vdots & \vdots & \ddots & \vdots \\
x_{n 1} & x_{n 2} & \cdots & x_{n N}
\end{array}\right]
$$

where $N$ represent the number of sensors placed on the bridge and $i^{\text {th }}$ column of matrix $A$ represents the vibration data of length $n$ collected from $i^{\text {th }}$ sensor. In the literature, PCA is used to find the eigenvalues and eigenvectors of the covariance matrix $A A^{T}$ to characterize the dynamics of the structure in reduced space. However, the computation of eigenvectors and eigenvalues are expensive if the length of the vibration data is large. To take care of the computational cost, Cavadas et al. have used moving principal component analysis (MPCA), which computes the eigenvectors and eigenvalues of covariance matrix of windowed submatrix of data matrix $A .{ }^{19}$

Using this approach, the characterization of varying environmental condition is difficult to model. On the other hand, Shane and Jha modelled the varying operational and environmental conditions by calculating the proper orthogonal modes (POM) of data matrices using the singular value decomposition (SVD). ${ }^{23}$ They have constructed a set of data matrices corresponding to different excitation (impact load) levels. The orthogonal modes/eigenvectors for each data sample matrix are calculated which makes the algorithm computationally expensive. Besides, the residual error between orthogonal modes of undamaged and damaged vibration data are used to detect and locate the damage in the structure. However, a good approximation of mode shape can only be obtained from the free or lightly damped vibration signals of a dense network. ${ }^{21}$ In this paper, PCA is used to model the undamaged vibration signals collected from multiple sensors under various environmental and loading condition in a computationally efficient way which makes the algorithm faster. Here, we model the various environmental and loading conditions considering environmental and loading variabilities are implicitly embedded in vibration signals. The proposed approach for damage detection and localization in truss bridge is described as follows. 
Suppose $A_{1}, A_{2}, \ldots, A_{k}, \ldots, A_{p}$ are set of data samples collected in $p$ different environmental and loading conditions constitute a training set, where $k$ corresponds to the environmental and loading condition under which the vibration data have been collected. The columns of data sample $A_{k}$ are concatenated and centred to zero mean to form a training vector $B_{k}$ for $k=1,2, \cdots, p$. All the training vectors are arranged in columns to form a training matrix $M$ like in Eq. (3).

To construct a model space, matrix $M$ is decomposed into score matrix $M^{*}$ and loading matrix $U$ using principal component analysis (PCA) as:

$$
M=M^{*} \cdot U^{T}
$$

where,

$$
U=\left[\begin{array}{llll}
u_{1} & u_{2} & \cdots & u_{p}
\end{array}\right] .
$$

The columns of the matrix $U$ serve as eigenvectors $u_{1}, u_{2}, \ldots, u_{p}$ corresponding to eigenvalues $\lambda_{1}, \lambda_{2}, \ldots, \lambda_{p}$ of the covariance matrix $\Sigma=M^{T} M$ such that $\lambda_{1} \geq \lambda_{2} \geq \cdots \geq$ $\lambda_{p}$. In linear algebra, it can be easily verified that eigenvalues of matrix $M^{T} M$ are also the eigenvalues of the matrix $M M^{T}$ wherever eigenvectors of $M^{T} M$ and $M M^{T}$ are strongly related. ${ }^{26}$ If $u_{i}$ is an eigenvector of $M^{T} M$ then $M u_{i}$ will be the eigenvector of $M M^{T}$. In this paper, the eigenvalues and eigenvector pair are obtained from the covariance matrix $M^{T} M$ instead of $M M^{T}$. After that, $M^{*}=M U$ is calculated to find the eigensamples where $M^{*}$ is our model space. The matrix $M^{*}$ can be viewed as the rotated version of training matrix $M$ in the eigenspace $U$. This approach characterizes the different operational and environmental conditions more efficiently and also preserves the useful information in reduced space. The columns of the matrix $M^{*}$ are normalized which constitute a model space, where $k^{\text {th }}$ column corresponds to the eigensample in the direction of $u_{k}$. Each training vector $B_{k}$ can be represented as the weighted sum of the $p$ eigensamples in the model space. Therefore, weighted feature vector corresponding to $k^{\text {th }}$ training vector $B_{k}$ is obtained as:

$$
\mathrm{v}_{k}=M^{* T} \cdot B_{k} .
$$

This weight feature vector uniquely defines the dynamics of the training data sample. Similarly, the weighted feature vectors $\mathrm{v}_{1}, \mathrm{v}_{2}, \ldots, \mathrm{v}_{p}$ for training data samples $A_{1}, A_{2}, \cdots, A_{p}$ are obtained respectively.

In the model space, the weighted test feature vector for an unknown test data sample $A_{\text {test }}$ is obtained using Eq. (7).

$$
\mathrm{v}_{\text {test }}=M^{* T} \cdot B_{\text {test }} ;
$$

where, $B_{\text {test }}$ is the test vector constructed from the test data sample $A_{\text {test }}$ as similar to $B_{k}$ in Eq. (2). It is worth noting that feature vector $\mathrm{v}_{\text {test }}$ defines the dynamics of the whole structure in terms of various environmental and loading conditions in the model space. The Euclidean distance between test feature vector and all training feature vectors are computed as :

$$
d_{i}=\left\|\mathrm{v}_{i}-\mathrm{v}_{\text {test }}\right\| \quad \text { for } i=1,2, \cdots, p .
$$

A training data sample closest to the test data sample is selected for having minimum distance in the model space, against which the test data sample is to be compared. This ensures that the test data sample and closest model data samples are collected in similar environmental and loading condition. If the test data sample $A_{\text {test }}$ is closest to $j^{\text {th }}$ training data
Table 1. Model parameters for a typical truss bridge structure.

\begin{tabular}{|l|c|}
\hline Length of the Bridge & $36.0 \mathrm{~m}$ \\
\hline Height of the Bridge & $7.0 \mathrm{~m}$ \\
\hline Width (Horizontal spacing) & $5.25 \mathrm{~m}$ \\
\hline Poisson's Ratio & 0.3 \\
\hline Modulus of Elasticity* & $2 \times 10^{11} \mathrm{~N} \mathrm{~m}^{-2}$ \\
\hline Mass Density & $7850 \mathrm{Kg} \mathrm{m}^{-3}$ \\
\hline * at room temperature $\left(20^{\circ} \mathrm{C}\right)$ \\
\hline
\end{tabular}

sample $A_{j}$ in model space then residual standard error $R_{i, \text { test }}$ for test data sample is computed in original space at $i^{\text {th }}$ sensor location as:

$$
R_{i, \text { test }}=\left|\sigma_{i, \text { test }}-\sigma_{i, j}\right|
$$

where $\sigma_{i, j}$ and $\sigma_{i, t e s t}$ are standard deviation of $A_{j}$ and $A_{\text {test }}$ respectively at $i^{t h}$ sensor location.

The local damage index (LDI) at $i^{\text {th }}$ sensor location is obtained by normalizing the residual standard error with standard deviation of closest undamaged data sample at the $i^{\text {th }}$ sensor location.

$$
\mathrm{LDI}_{i}=\frac{R_{i, t e s t}}{\sigma_{i, j}} .
$$

If the unknown test data sample is collected from the undamaged bridge, then LDI approaches zero. On the other hand, the LDIs increase significantly as damage over the structure increases. Damage severity of the whole structure is obtained by accumulating LDIs corresponding to all sensors placed on the structure as damage index (DI), as in Eq. (11).

$$
\mathrm{DI}=\sum_{i=1}^{N} \mathrm{LDI}_{i}
$$

In the proposed approach, finding the closest model data can be viewed as a data normalization under varying operational and environmental conditions in SHM. ${ }^{27}$ For the data normalization, it is necessary to have all source of variability. ${ }^{11}$ Therefore the proposed model needs to be robust, and the training samples must be collected in all sources of variability so that varying environmental and loading conditions will be well characterized by the training samples in the model space. If the training samples are characteristic of a limited range of operational and environmental variations then the accurate model may not be modelled to characterize the varying environmental and loading conditions.

\section{NUMERICAL CASE STUDY}

\subsection{Numerical Model}

The efficacy of the proposed algorithm is tested on simulated acceleration data samples obtained from a finite element model of a steel truss bridge structure modelled in ANSYS Workbench 15.0 as shown in Fig. 1.

The finite element (FE) method is a numerical method used to solve problems of engineering and mathematical physics. ${ }^{28}$ Beam188 element is used to model the structure which includes shear deformation based on the Timoshenko beam theory. The material properties and geometrical parameters of a typical single lane railway truss bridge model of effective span length of $36 \mathrm{~m}$ are provided in Table $1 .{ }^{29}$

The structure consists of truss members having different cross sections as per design. ${ }^{29}$ The constraint applied to the structure is very similar to a real bridge structure. The left 


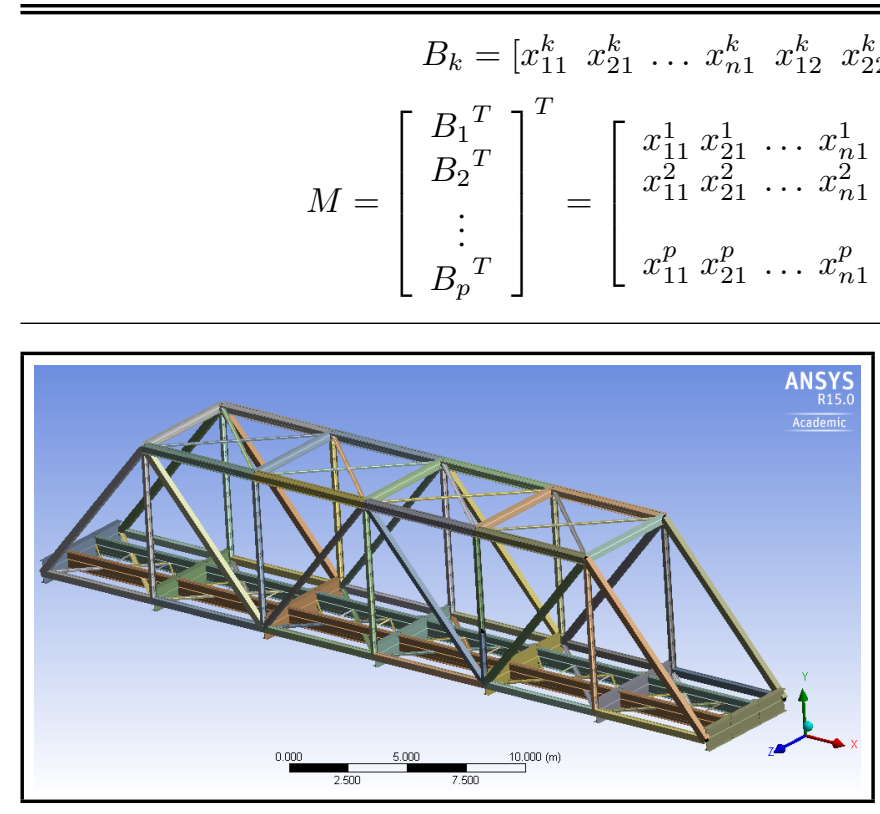

Figure 1. Steel truss bridge structure modelled in ANSYS workbench.

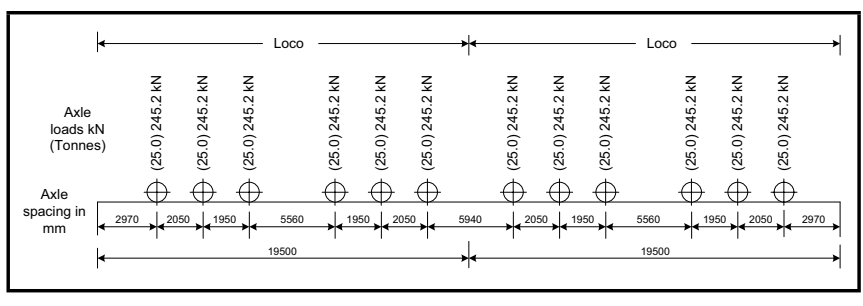

Figure 2. Broad gauge loading model.

end of the bridge model is pin-supported and has roller support at the right end. All the truss member joints are modelled as welded joints. The structure is composed of steel members meshed to form a rigid frame as shown in Fig. 1.

To excite the structure, a moving locomotive axle load is applied on the stringer at a constant speed, $v=L / \tau$, where $\tau$ is the traveling time across the bridge and $L$ is the length of the bridge structure. Here, the assumption is made that the locomotive is moving at the constant speed which can be easily controlled by a locomotive pilot during the observation period of the health monitoring. The spacing between the axle loads are according to the broad-gauge locomotive load model as shown in Fig. 2.30 The damping ratio of a steel truss bridge structure is typically set as $2 \%$ during the simulation. ${ }^{31}$ In our study, we have performed a full transient analysis allowing all the nodes to move in all six degrees of freedom (DOF) to obtain more realistic time-history data.

\subsection{Data Simulation}

The acceleration time-history signals are measured from 20 different sensor locations (S1-S20) placed on the web joints of the truss bridge structure as shown in Fig. 3. The sensor locations are decided based upon the concept that the moving locomotive loads acting on the stringer redistribute to other truss members through the web joints. Acceleration signals are captured from all sensor location for 5 seconds at the sampling frequency of $200 \mathrm{~Hz}$ resulting in 1000 samples of data recorded in individual sensors. Structural vibration, while the moving locomotive axle load acts on the structure, is considered for the damage diagnosis. The broad gauge locomotive having an axle

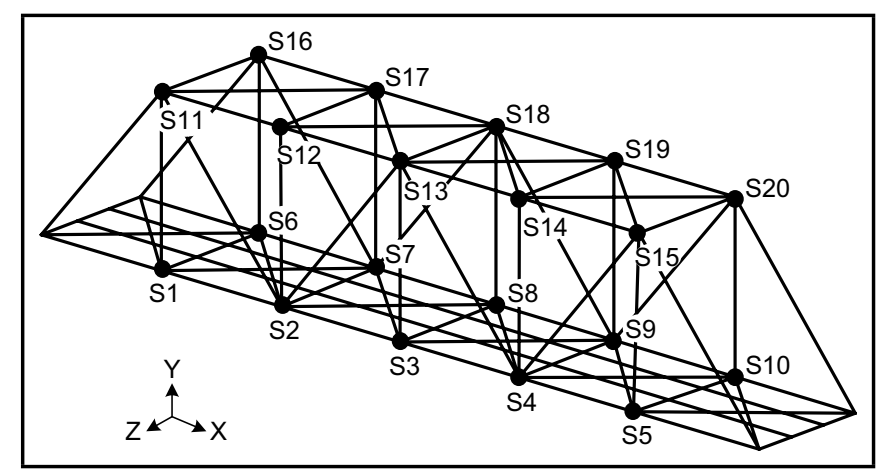

Figure 3. Sensor locations for acceleration time-history data collection.

load $245.2 \mathrm{kN}$ (as per Indian Railway Bridge Rule ${ }^{30}$ ) is considered for excitation as shown in Fig. 2. The chosen locomotive axle load is equivalent to a locomotive load of 25 tons. However, the locomotive axle load does not change the net mass of the structure. To consider the varying load, 29 different locomotive axle loads, varied in the range of $245.2 \pm 20 \% \mathrm{kN}$, are considered for the data simulation. In this work, changes in temperature are considered as environmental variations. Temperature variation was modelled as the change of modulus of elasticity of the steel material according to Poh model. ${ }^{32}$ In the simulation, the coefficient of the expansion of the steel is considered as $11.7 \times 10^{-6}$ per $1^{\circ} \mathrm{C} .{ }^{30}$ The temperature variation in the range of $0-50{ }^{\circ} \mathrm{C}$ at an equal interval of $5{ }^{\circ} \mathrm{C}$ was considered in the simulation. It is worth noting that the equal interval is considered for the sake of simplicity. One can choose the random distribution of the temperature in the range for the data simulation. The objective is to measure structural responses in various operational and environmental conditions. We have simulated all combination of locomotive axle loads and temperature variations, without considering any damage for generating the training data set. In a real-world application, training samples must be collected in all sorts of various operational and environmental conditions. That can be measured by multiple passes of the locomotive with various loads at different instances of time during the training period. In the simulation, all the combinations of different loads and temperatures give 319 healthy data samples constituting a training set (Set I), which are used for the creation of model space $M^{*}$. Set II has both healthy and damaged data samples collected in various environmental temperature and loading conditions different from Set I but in the specified range. Healthy data samples (HS) in Set II are not included in the training set (Set I). Healthy data samples in Set II are simulated to decide the threshold of discrimination between healthy and damaged structural conditions. Damaged data samples (DS) in Set II are simulated by reducing the stiffness of the web member by $10 \%, 20 \%$ and $30 \%$ in various environmental and loading conditions. To consider the measurement and ambient noise, the simulated data is polluted with white Gaussian noise. In many applications of SHM, 1-5\% white Gaussian noise is consid- 


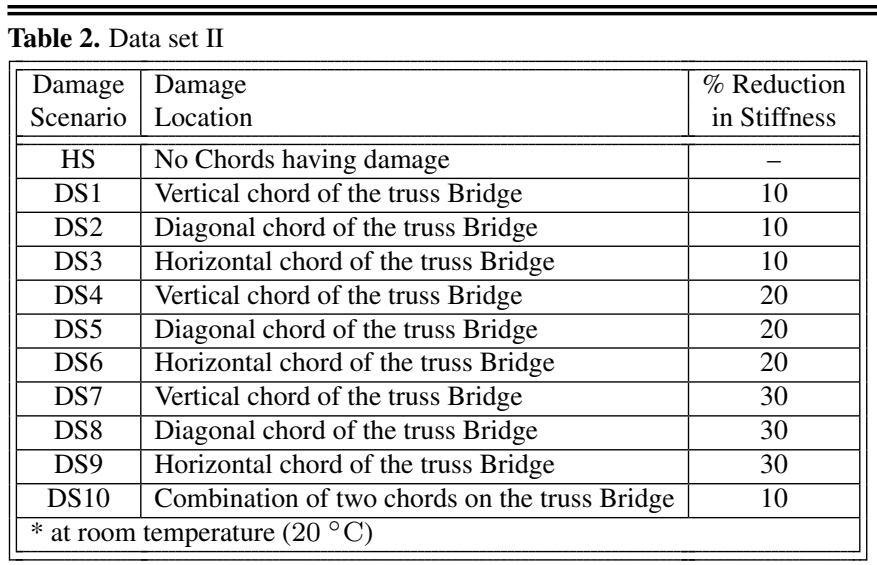

ered to pollute the simulated vibration signals to consider the measurement and ambient noise. ${ }^{15,33}$ Shu et al. have considered 2-5\% white Gaussian noise to the moving load response of the simplified railway bridge model. ${ }^{15}$ In this work, $2-5 \%$ white Gaussian noise with zero mean and specified standard deviation are added to the vibration signals. The sensitivity of the proposed algorithm towards the noise is tested by adding $0 \%$ (no noise), $2 \%$, and $5 \%$ white Gaussian noise. The noise contaminated signals are obtained as:

$$
\mathrm{x}_{n}=\mathrm{x}_{c}+e_{p} N_{0} \sigma\left(\mathrm{x}_{c}\right)
$$

where $\mathrm{x}_{c}$ is simulated signal without any noise; $\mathrm{x}_{n}$ is the polluted signal. The set $e_{p}$ is the percentage of noise to be added before processing the signal which is the ratio of standard deviation value between noise and signal. The set $N_{0}$ is the standard normal distribution, and $\sigma\left(\mathrm{x}_{c}\right)$ is the standard deviation of $\mathrm{x}_{c}$. The polluted vibration signals corresponding to sensor S8 before and after damage with their power spectrum are shown in Fig. 4.

In Table 2, the damage scenario considers various damage locations which include 10 vertical chords, 12 diagonal chords, and 20 horizontal chords for the data simulation. Three levels of damage for each location are simulated to test the sensitivity of the proposed DI toward the severity of damage.

DS1-DS9 are for single damage (stiffness reduced for a single chord) on the structure. In which, DS1-DS3, DS4-DS5, and DS6-DS9 are for damage levels with 10\%, 20\%, and 30\% reduction of stiffness, respectively. DS10 is for double damage. In this case, stiffness of two truss members are reduced simultaneously by $10 \%$.

\section{RESULTS AND DISCUSSION}

Vibration signals collected under various environmental and loading conditions are processed through the proposed algorithm. The data samples $A_{k}$ is a $1000 \times 20$ dimensional matrix i.e., $n=1000$ and $N=20$. All the healthy data samples from Set I are used to construct the training matrix $M_{20000 \times 319}$ which constitute a model space $M^{*}$ using PCA as discussed in Section 2. In the model space, 319-dimensional weighted feature vectors corresponding to all undamaged and damaged data sample are computed. The weighted feature vector corresponding to the test data sample are used to find the closest match to the undamaged data sample from Set I. Further, the residual standard error is computed and normalized to obtain the LDI at each sensor location using Eqs. (9) and (10). Undamaged data samples in Set II are used to determine the threshold value for the DI to distinguish between healthy and damaged bridge conditions. Damage scenarios, DS1-DS10, in Set II are used for testing purpose. The damage diagnosis results for various damage scenarios are discussed in following subsections.

\subsection{Damage Detection}

In this section, detection and localization of damages are performed for various damage scenarios. Damage indices (DIs) are calculated for damaged and undamaged bridges. Ideally, the threshold value of the DI for the undamaged bridge should be zero, but due to random variation in the environmental change, it deviates from zero. To discriminate between undamaged and damaged bridge conditions, the outlier statistic is used. Once the DI value exceeds the threshold value, the corresponding test data sample is classified as a damaged data sample, otherwise undamaged. The threshold value is decided by constructing an X-bar control chart using the $\overline{\mathrm{DI}}$ and $\sigma$ as the mean and standard deviation of the DIs corresponding to the healthy data samples in Set II. ${ }^{34}$ The X-bar control chart, centre line CL, lower control limit (LCL), and upper control limit (UCL) are defined as :

$$
\mathrm{CL}=\overline{\mathrm{DI}}, \quad \mathrm{LCL}=\overline{\mathrm{DI}}-\alpha \sigma, \text { and } \mathrm{UCL}=\overline{\mathrm{DI}}+\alpha \sigma ;
$$

where $\alpha$ defines the confidence interval over the number of undamaged data samples. In this case study, $\alpha$ is considered 3 for a confidence interval of $99.7 \%$, which allows for how many undamaged data samples that can be considered as outliers. ${ }^{35}$ For the simulated data, the UCL is obtained as 0.016 which is considered as the threshold to distinguish between undamaged and damaged structural conditions.

The LDI corresponding to the individual sensor locations of damaged structure indicate the local damage severity. A local threshold is defined to locate the damage in the structure. As mentioned earlier, the damage in different truss members have different impacts on the structure. Therefore, the LDI is normalized with the corresponding global DI of the structure, so that each LDI is proportional to its original value (the value will be between 0 and 1) and the sum of all values is 1 . The normalized LDI for a few damage scenarios with $2 \%$ noise, from Set II are shown in Fig. 5. It was observed from the collected vibration data that if normalized LDI exceeds 0.15 (15 $\%$ of DI) for any sensor location, then that sensor location is identified as the damage location. It is worth noting that the threshold value is sensitive to the number of sensors placed on the monitoring structure. As the number of sensors increase the local threshold value will reduce.

The damage detection and localization colour map for the same damage scenarios with $2 \%$ noise are shown in Fig. 6 . The local damage in the truss bridge structure indicated by dotted line truss members are shown in the left column of the Fig. 6. The colour map image shows the LDI at corresponding sensor locations and their damage localization in the corresponding entry in the right column. Each colour map is obtained by interpolating the LDI of each sensor. In truss bridge structure, different members play a different role to give strength to the structure. Some chords are subjected to tension, and some chords are subjected to compression during excitation. Therefore, damage to vertical, diagonal, and horizontal chords are investigated separately. The color map for a healthy bridge 


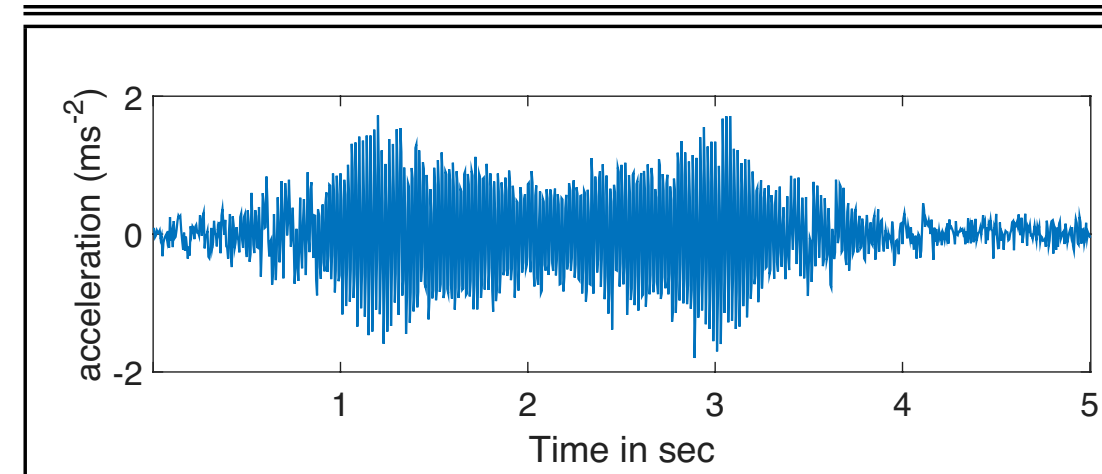

(a)

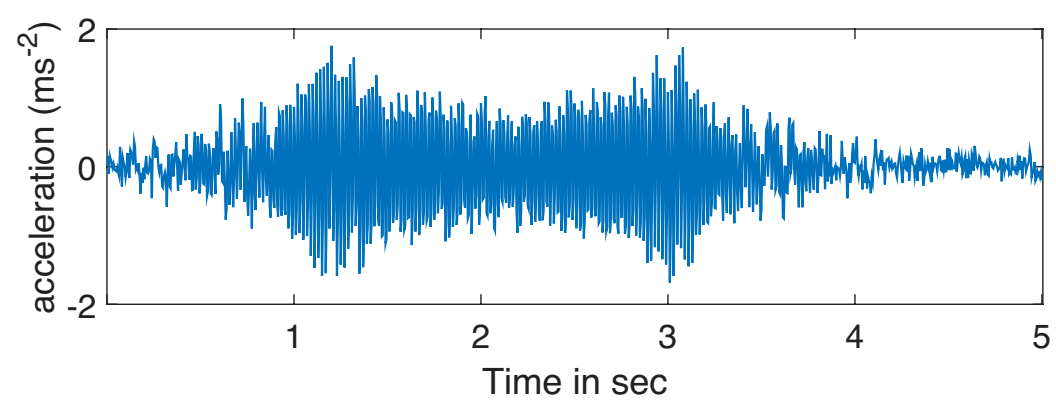

(c)

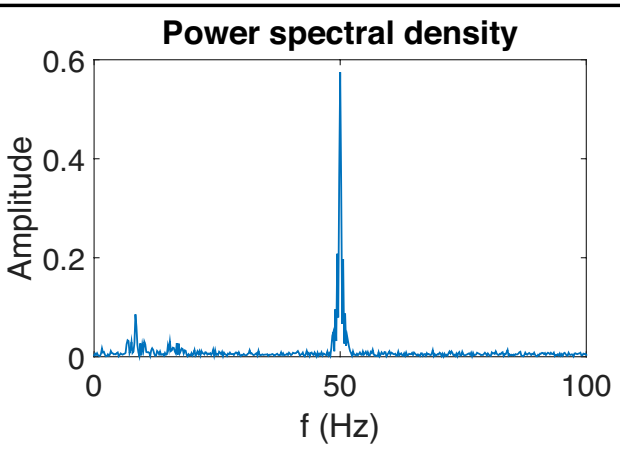

(b)

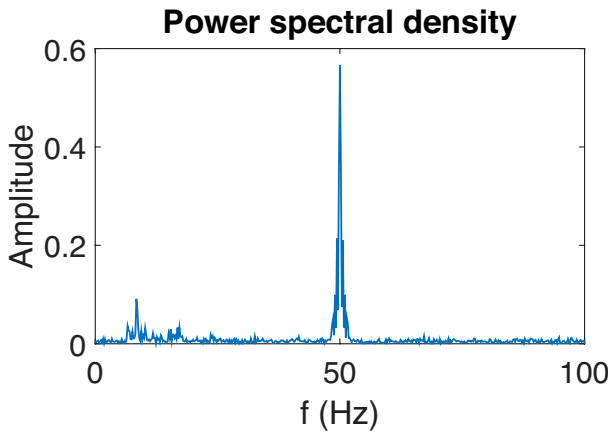

(d)

Figure 4. Vibration signals collected from sensor S8 with $2 \%$ noise and corresponding power spectral density: (a) \& (b) before damage and (c) \& (d) after damage

scenario is shown in Fig. 6a. It can be observed from the corresponding normalized LDI in Fig. 5a that all the normalize LDIs are below 0.15 . Figure $6 \mathrm{~b}$ is for damage detection in vertical chords between sensor S4-S14 (damage scenario DS7). It can be observed that the maximum LDI (exceeds 0.15 in Fig. 5b) is obtained at sensor location S14 that is placed at one end of the damaged chord. On the other hand, the LDI at the sensor location S4 is not very high. One of the reasons may be that diagonal members additionally support the web joint at sensor location S4. Similarly, Figs. 6c and 6d represent damage scenarios where a diagonal member between sensor S9-S18 (damage scenario DS8) and a horizontal member between sensor S2-S3 (damage scenario DS9) respectively, are damaged. The LDIs corresponding to vertical, diagonal, and horizontal members show that damages in diagonal members of truss structures are more critical and demand special attention during health monitoring. Furthermore, it justifies that each member in a truss structure behaves differently for the same level of damage. Brunell et al. ${ }^{24}$ and Kim et al. ${ }^{12}$ have also drawn the similar conclusion. Two damages at different locations (damage scenario DS10) are also tested as shown in Figs. 6e and $6 \mathrm{f}$. The results obtained show that both the damage locations are localized with $15 \%$ change in the LDI (Figs. 5e and 5f). It is worth nothing that the LDI at one end of the damaged member is very high which indicates the location of the damage. However, the proposed algorithm is unable to identify the exact damaged member in the structure. It can only identify the sensor location near truss member experiencing damage.

There are few cases with damage at two different locations; the algorithm detects a single damage because for the similar level of damage two different members behave differently. For example, a $10 \%$ stiffness reduction in a vertical member is not equivalent to $10 \%$ stiffness reduction in a diagonal member. This may be the reason that in Fig. 6f only one damage loca- tion is clearly visible wherever another damage location is not clearly visible. It is expected that after the rehabilitation of the damaged diagonal member, damage in vertical member will be clearly visible.

\subsection{Damage Assessment For Various Levels of Damages}

To detect the damage severity of the structure for various levels of damages, we have tested different damage scenarios DS1-DS9. The same vertical, diagonal and horizontal damaged members taken in the previous section are investigated for three different levels of damages. Figures 7, 8 and 9 show the damage localization with respect to the sensor locations for various damage scenarios.

The LDIs are shown in Fig. 7 for three different levels of damages $(10 \%, 20 \%$, and $30 \%$ stiffness reduction) for a damaged vertical member between sensors S4-S14. It can be observed that the LDI corresponding to sensor S14 increases as local damage over the structure increases. Similar outcomes are observed in Figs. 8 and 9 for diagonal and horizontal members, respectively.

\subsection{Damage Assessment Under Various Environmental and Loading Conditions}

The effectiveness of the proposed algorithm under different environmental temperature and loading conditions is also investigated with two different noise levels (2\% and 5\% noise). The LDI at environmental temperatures $\mathrm{T} 1\left(20{ }^{\circ} \mathrm{C}\right)$ and $\mathrm{T} 2$ $\left(40{ }^{\circ} \mathrm{C}\right.$ ) are obtained for two randomly selected loads (L1 and L2) in the range of $245.2 \pm 20 \% \mathrm{kN}$. It is observed that the LDI due to different excitation at different environmental temperatures is similar to the same label of damage as shown in Figs. 10 and 11. This establishes the robustness of our damage 


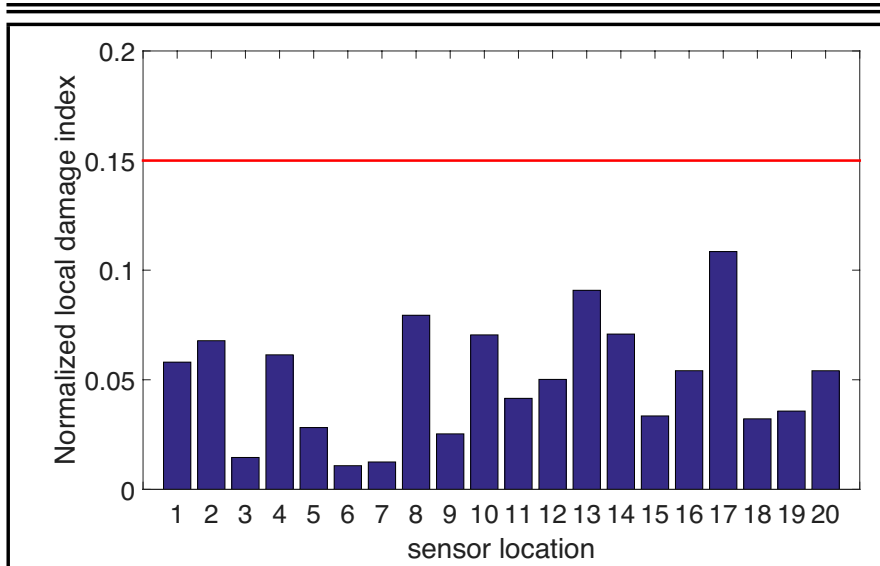

(a)

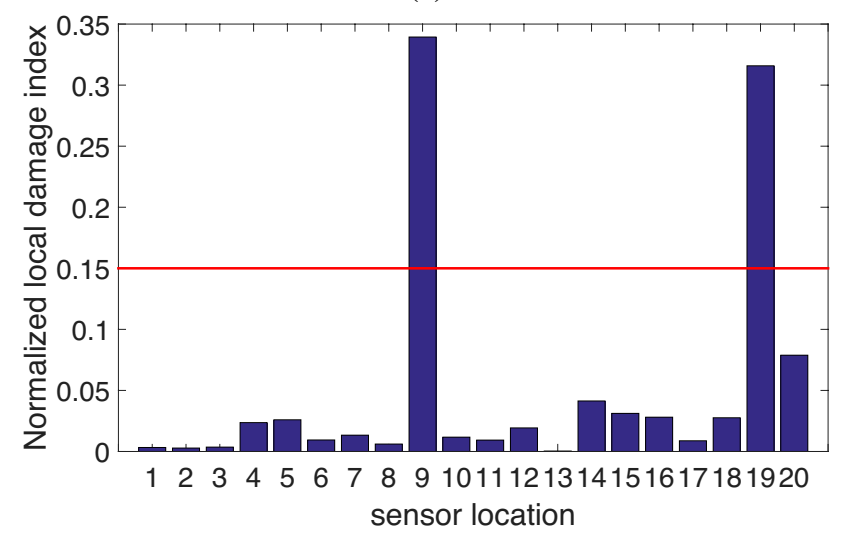

(c)

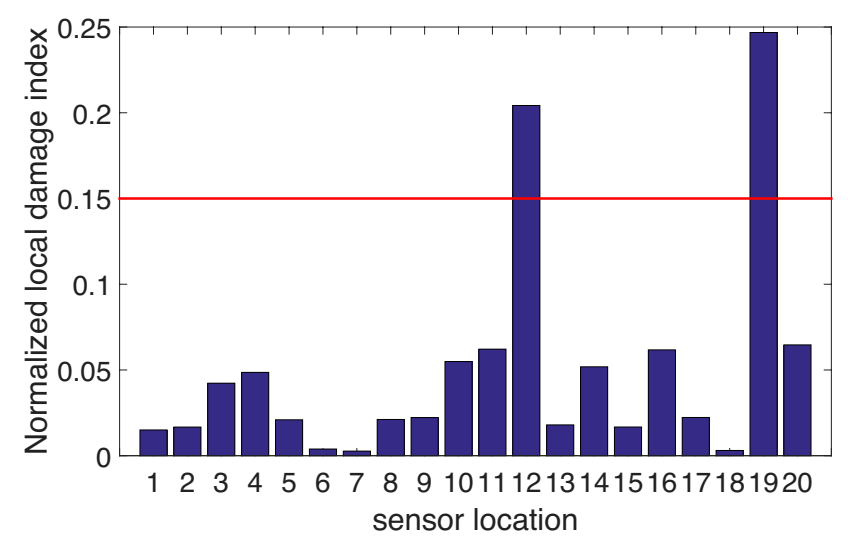

(e)

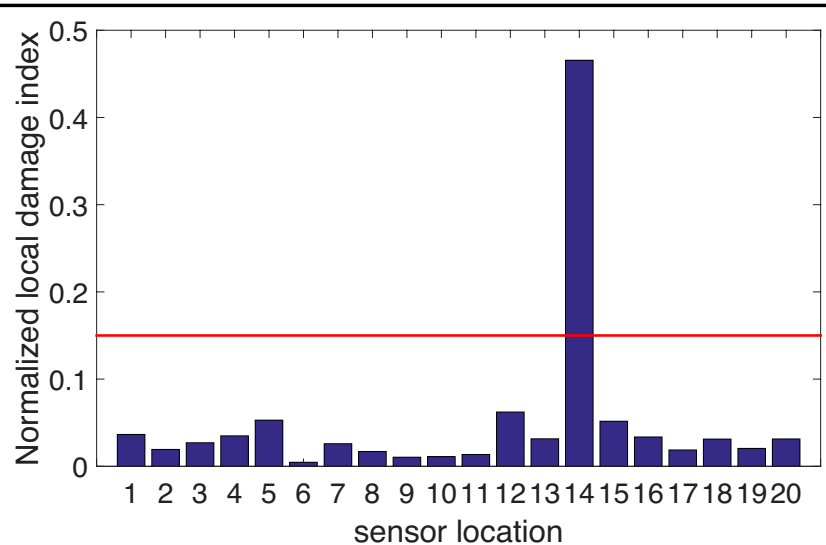

(b)

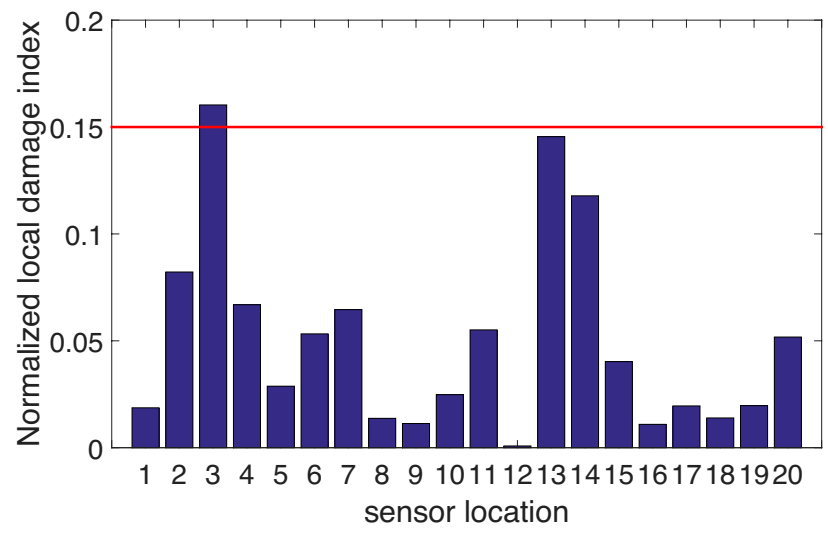

(d)

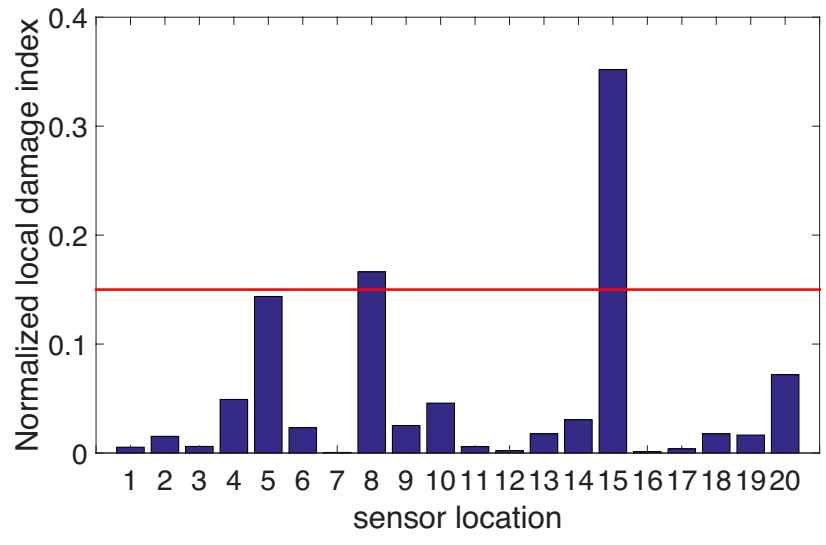

(f)

Figure 5. Normalized local damage index for various damage scenarios with $2 \%$ noise: (a) no chords having damage (HS), (b) single vertical chord damaged between sensor S4-S14 (DS7), (c) single diagonal chord damaged between sensor S9-S18 (DS9) (d) single horizontal chord damaged between sensor S2-S3 (DS8), (e)-(f) two chords damaged at different location (DS10).

detection algorithm against environmental and load variations. This is due to the normalization of the residual standard error with respect to the model data closest to the test data as in Eq. 10.

\subsection{Sensitivity of the Proposed Algorithm Towards Noise}

In this section, sensitivity of the proposed algorithm is tested for three different noise levels $(0 \%, 2 \%$, and $5 \%)$. The LDI obtained for three damage scenarios, DS1, DS4, and DS7 are shown in Fig. 12 for three different noise levels. It can be observed that the proposed algorithm easily localizes the highlevel damage, even in the case of 5\% noise. However, the lowlevel damage is more sensitive to noise. The $5 \%$ noise masked the change in vibration signal due to the low-level damage where it is hard to localize the damage.

\subsection{Comparative Analysis of the Proposed Algorithm with POD Based Algorithm}

We have compared our proposed method with the method of Shane and Jha for quantitative analysis. ${ }^{23}$ They have developed an algorithm for damage detection and localization based on POD for a cantilever plate after applying an impact load. The same algorithm was applied on the truss bridge structure using the same methodology, except the excitation to the structure. The structure was excited with a moving load instead of applying a load at a single point. For the damage diagnosis using POD, we have created two healthy data sets by splitting 


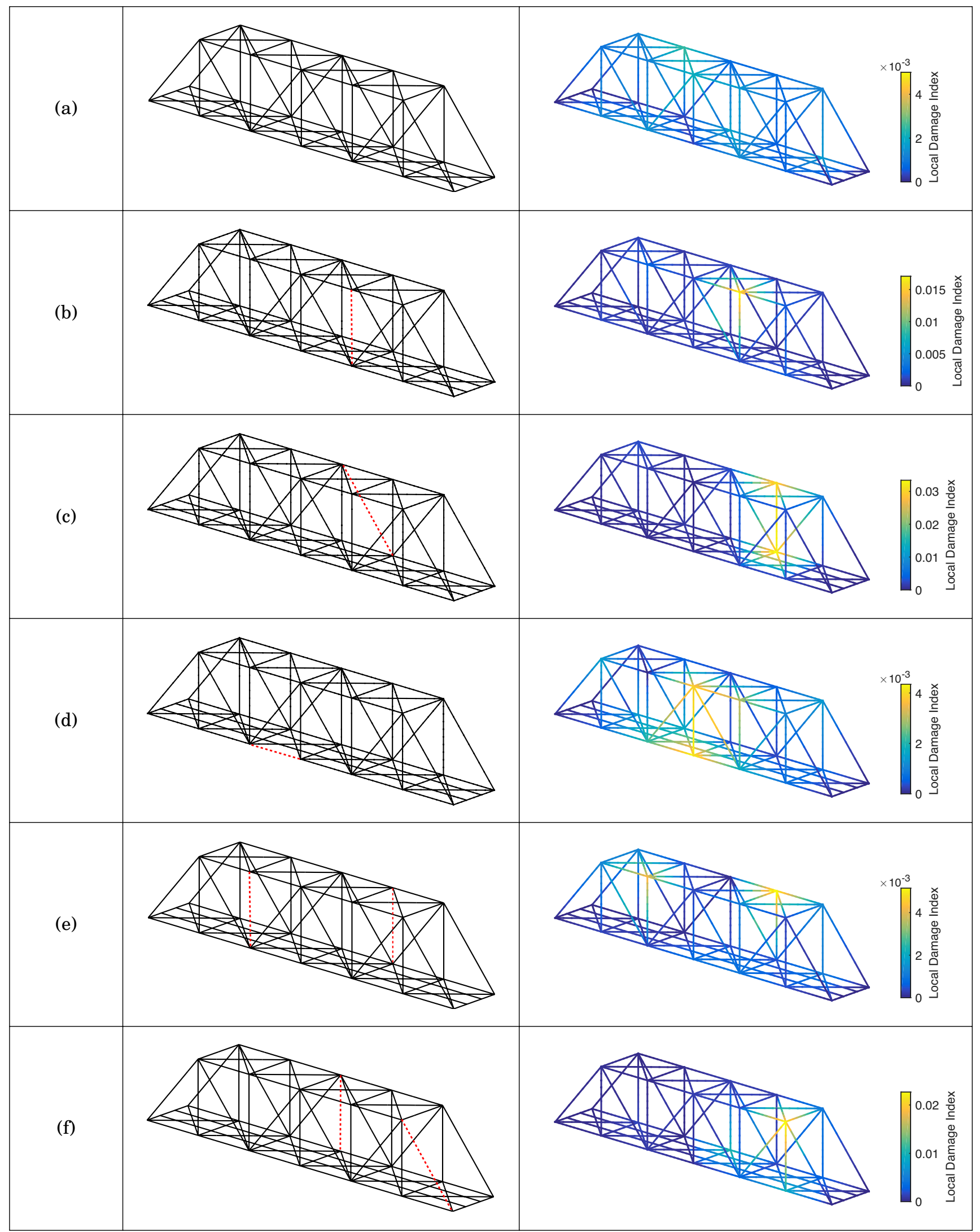

Figure 6. Local damage index colour map for damage detection and localization for various damage scenarios with $2 \%$ noise: (a) no chords having damage (HS), (b) single vertical chord damaged between sensor S4-S14 (DS7), (c) single diagonal chord damaged between sensor S9-S18 (DS9) (d) single horizontal chord damaged between sensor S2-S3 (DS8), (e)-(f) two chords damaged at different location (DS10). 


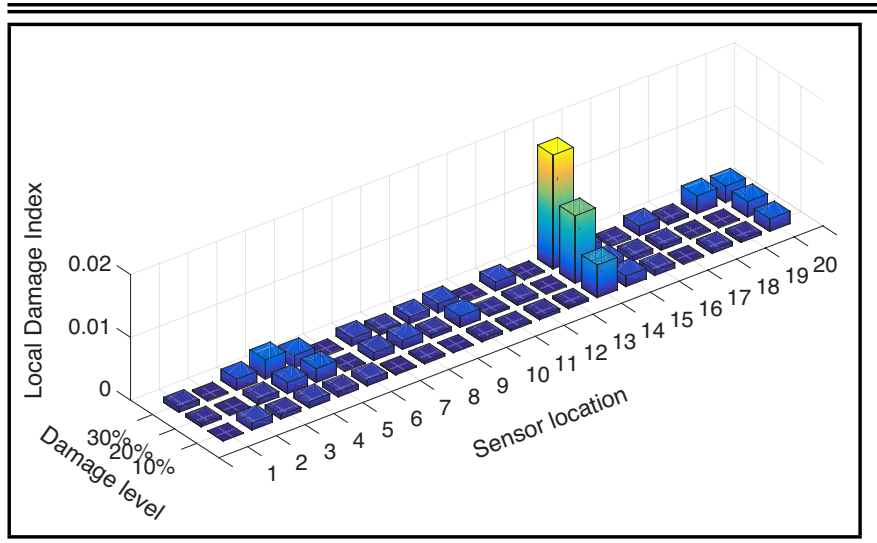

Figure 7. Local damage index corresponds to individual sensors for damaged chord between sensors S4-S14.

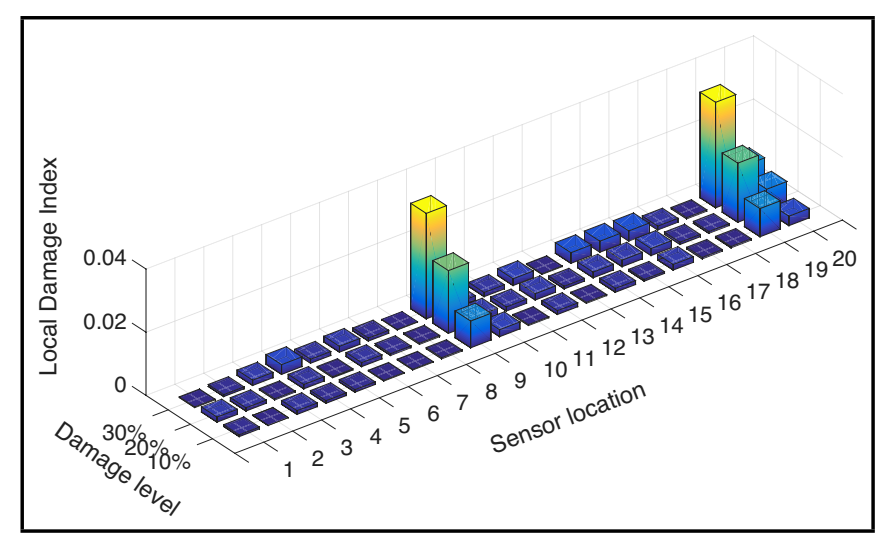

Figure 8. Local damage index corresponds to individual sensors for damaged chord between sensors S9-S18.

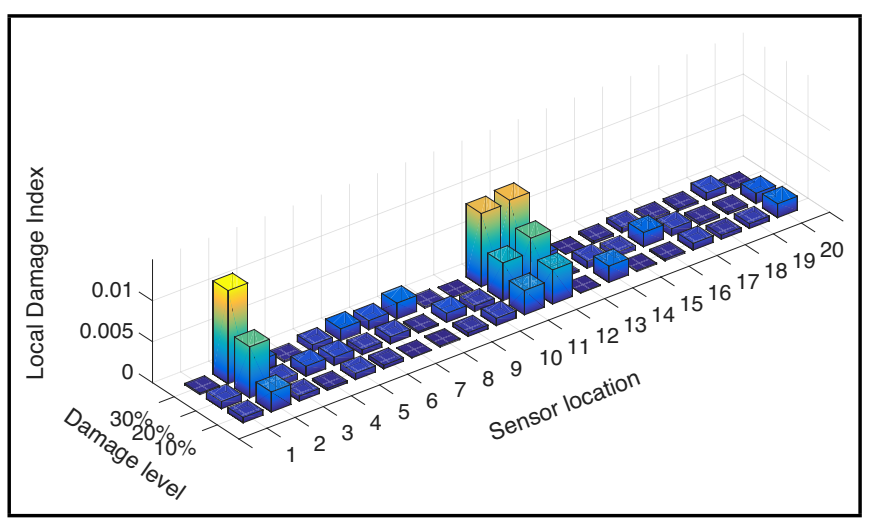

Figure 9. Local damage index correspond to individual sensors for damaged chord between sensors S2-S3.

the training data set (Set I) in two sets. Orthogonal modes of all the healthy data sets were identified using singular value decomposition (SVD). A test data sample was used to find the closest healthy sample from the first healthy data set by comparing their orthogonal modes. SVD was used to find orthogonal modes to find the closest healthy sample from the first healthy data set. Residual error was calculated between the closest healthy data sample and the projection of test data sample on the orthogonal modes of closest healthy data sample. It is difficult to get accurate orthogonal modes using a limited number of sensors for the truss structure, which makes the algorithm insensitive to small damage in the structure and gives a false alarm for damage in many cases.

In the contrast, we find the closest model data sample from

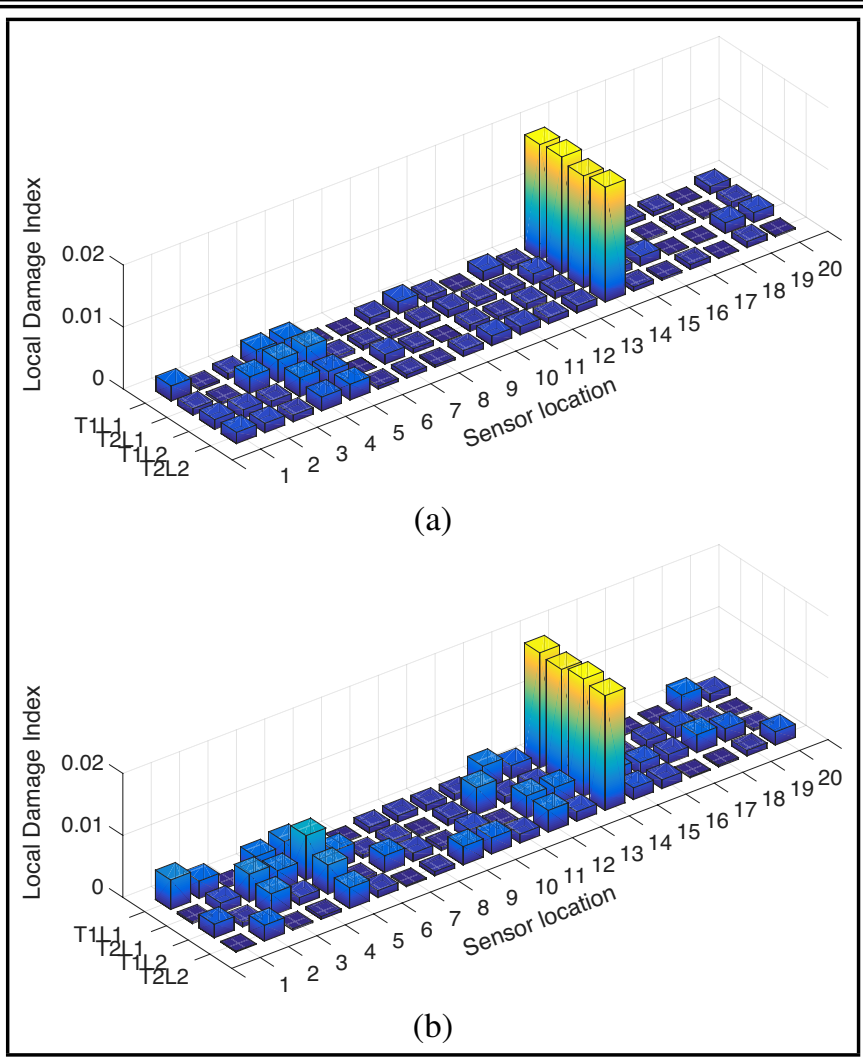

Figure 10. Local damage index for damage scenario DS7 at different temperature and loading conditions for same level of damage with (a) $2 \%$ noise, and (b) $5 \%$ noise

the training set based on the reduced size model in the model space using PCA. Afterward, the residual standard error was computed from the spread of data samples to find an effective damage index for all sensor locations using the proposed algorithm. The LDI corresponding to each sensor location and their colour map are shown in Fig. 13using these two methods. The damage index map was compared to vertical member damage (between sensor locations S4-S14) for three levels of damage. The LDI of both approaches were normalized for better comparison. It can be observed that our proposed algorithm is more efficient to localize the damage and more suitable for truss structures. The POD based algorithm is not capable of localizing the lower levels of damage as shown in Fig. 13a. In the case of high-levels of damage, the POD-based algorithm localizes the damage, but it assigned a higher local damage index for other sensor locations. On the other hand, our proposed algorithm is capable of localizing the damage for all levels of damage correctly, as shown in Figs. 13b, 13d, and 13f.

\section{CONCLUSION}

This paper presents a continuous health monitoring system for steel truss bridges under varying environmental temperature and load conditions. A numerical study has been performed to investigate the effect of local damage in steel truss members on the overall behaviour of the bridge. A typical railway steel truss bridge model is simulated, and structural responses are collected under a wide range of temperature and load conditions. The modulus of elasticity and coefficient of thermal expansion of the steel material are assumed to be temperature dependent. The modulus of elasticity is reduced for all members in the simulated bridge to consider temperature 


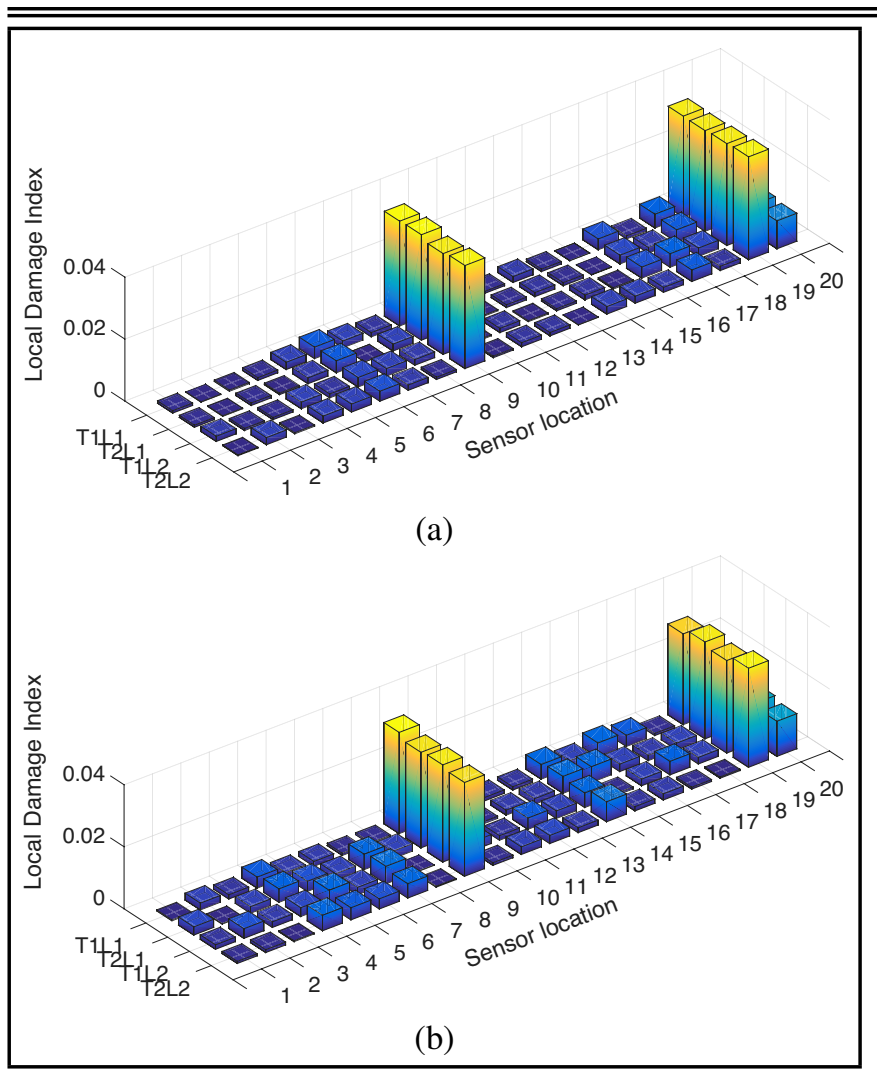

Figure 11. Local damage index for damage scenario DS9 at different temperature and loading conditions for same level of damage with (a) $2 \%$ noise, and (b) $5 \%$ noise.

variability in the range of $0-50{ }^{\circ} \mathrm{C}$. Damage to the structure is introduced by reducing the modulus of elasticity of different truss members.

A new methodology has been developed using PCA that characterizes the dynamic response of the structure in model space. The method has been validated by the moving load responses collected from the numerical model of the truss bridge. The developed methodology is capable of detection and localization of damage under a wide range of environmental and loading conditions. The presences of local damage in the truss bridge structure significantly influence the severity of the damage to the bridge. Various levels of damages were simulated and studied by varying the stiffness of different truss members. It has been observed that different truss members behave differently, even for the same level of damages. Truss bridge structures are more influenced by damage in diagonal members, compared to other members for the same degree of damage. The sensitivity of the proposed algorithm towards the different level of measurement and ambient noise has been studied. In the presence of a significant noise level, the proposed algorithm is unable to localize the low-level damage. A comparative analysis between a POD-based and proposed damage diagnosis approach has been presented. Experimental results show that our proposed algorithm is capable of detection and localization of damage with higher confidence under the same level of noise.

Further enhancement and testing are needed to validate the proposed algorithm under other varying environmental factors such as wind, humidity, etc. There may be situation where the inspection team wants to know the structural condition with the probable damage location in the structure with fewer sensors. In that situation, the proposed algorithm can perform effec-

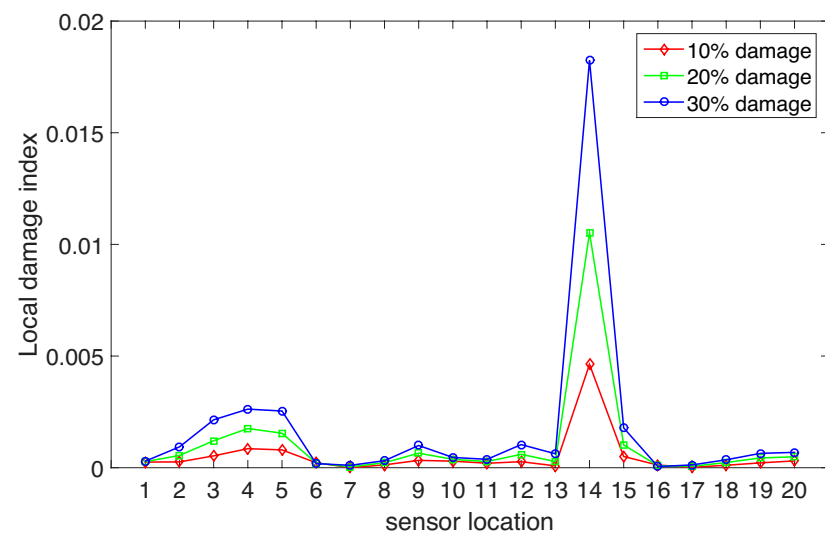

(q)

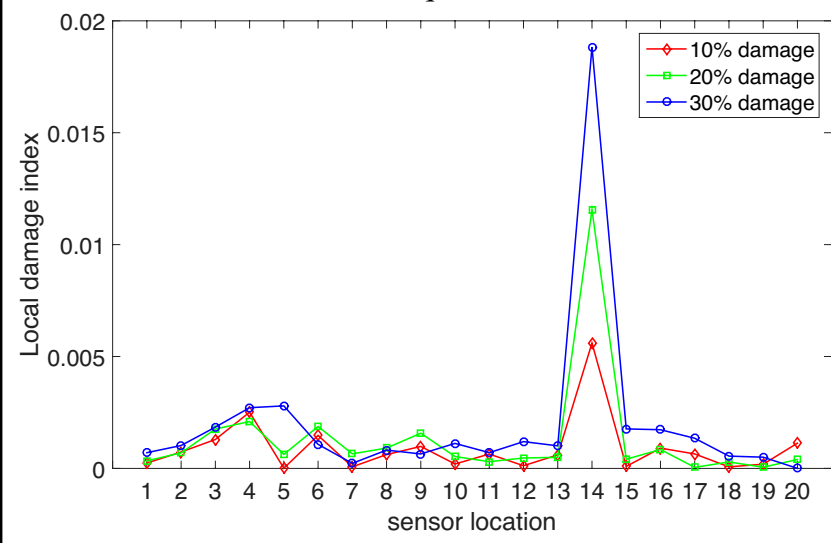

(b)

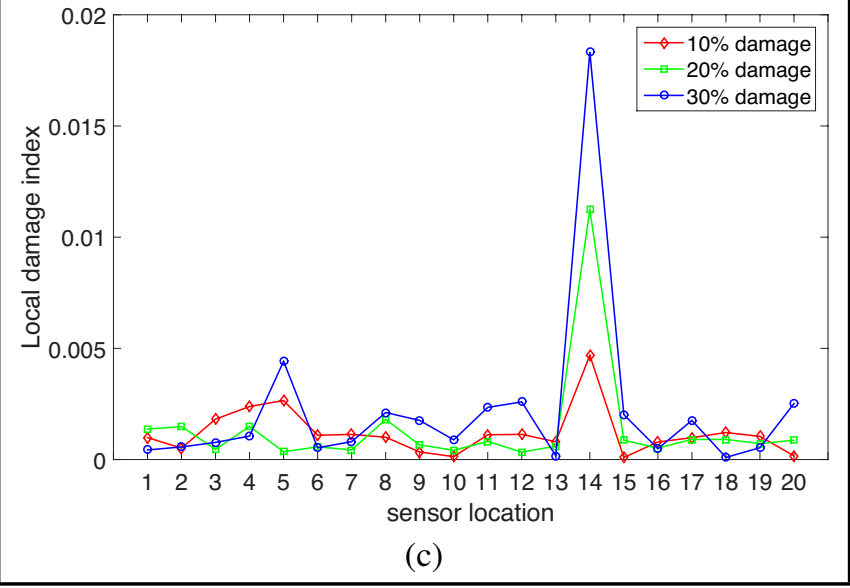

Figure 12. Local damage index for damage scenarios DS1, DS4, and DS7 in case of (a) $0 \%$ noise (no noise), (b) $2 \%$ noise, and (c) $5 \%$ noise.

tively. Our work is limited to localizing the damage by finding the sensor location near the damage. Further improvements are suggested to use correlation between a pair of signals to find the exact member under damage.

\section{REFERENCES}

1 Farrar, C. R. and Jauregui, D. A. Comparative study of damage identification algorithms applied to a bridge: I. experiment, Smart Materials and Structures, 7 (5), 704-719, (1998). https://dx.doi.org/10.1088\%2F0964$1726 \% 2 \mathrm{~F} 7 \% 2 \mathrm{~F} 5 \% 2 \mathrm{~F} 013$

2 Yeung, W. T. and Smith, J. W. Damage detection in bridges using neural networks for pattern recognition of vibra- 


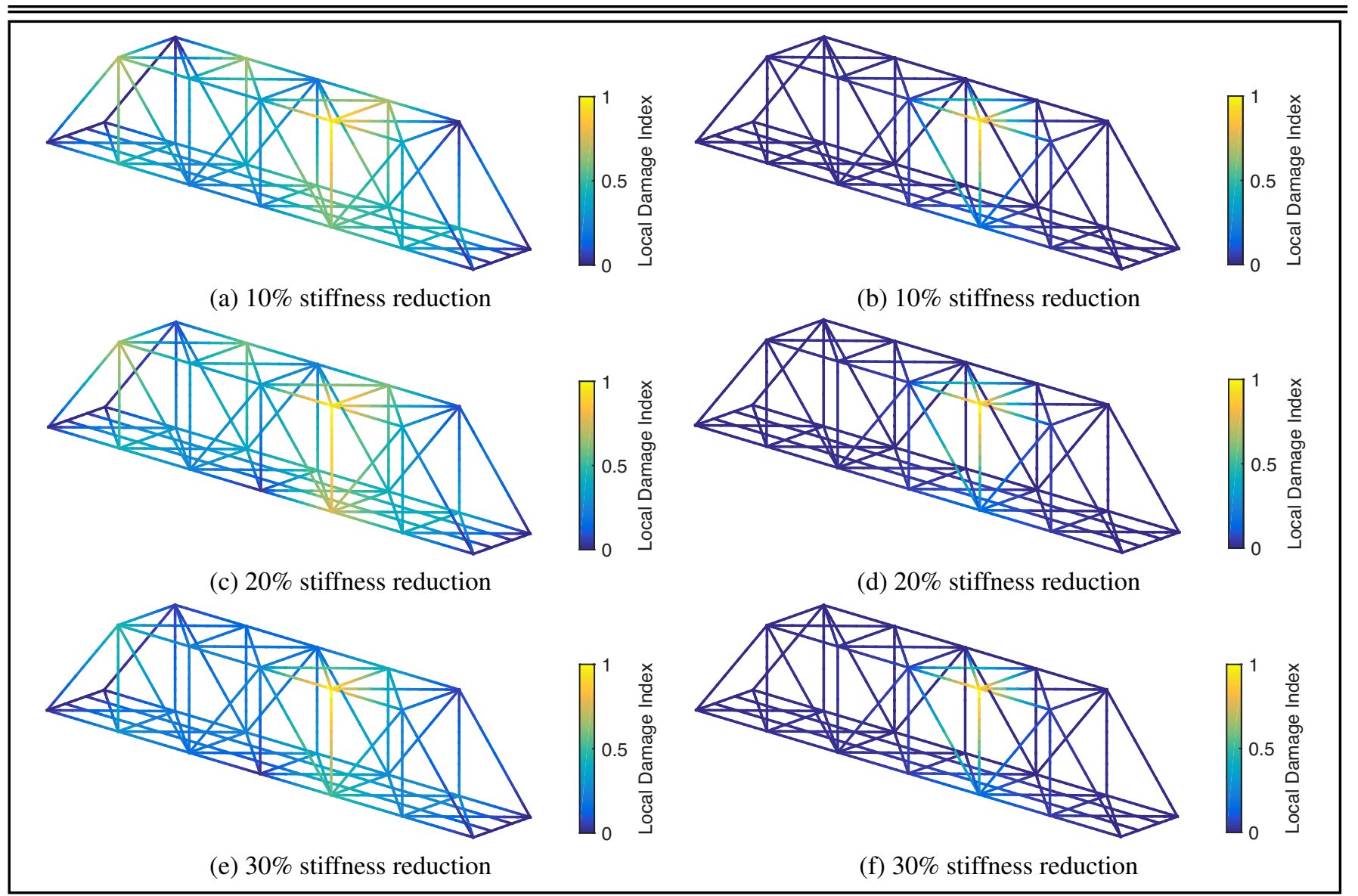

Figure 13. Damage localization for different levels of damages (truss member between sensor locations S4-S14) - using POD based approach (left column) and using proposed algorithm (right column).

tion signatures, Engineering Structures, 27 (5), 685-698, (2005). https://dx.doi.org/10.1016/j.engstruct.2004.12.006

3 Lee, J. J. and Yun, C. B. Damage diagnosis of steel girder bridges using ambient vibration data, Engineering Structures, 28 (6), 912-925, (2006). https://dx.doi.org/10.1016/j.engstruct.2005.10.017

$4 \mathrm{Xu}, \mathrm{Z} .-\mathrm{D}$. and $\mathrm{Wu}, \mathrm{Z}$. Energy damage detection strategy based on acceleration responses for long-span bridge structures, Engineering structures, 29 (4), 609-617, (2007). https://dx.doi.org/10.1016/j.engstruct.2006.06.004

5 Chang, K.-C. and Kim, C.-W. Modal-parameter identification and vibration-based damage detection of a damaged steel truss bridge, Engineering Structures, 122, 156-173, (2016). https://dx.doi.org/10.1016/j.engstruct.2016.04.057

6 Doebling, S. W., Farrar, C. R. and Prime, M. B. A summary review of vibration-based damage identification methods, Shock and Vibration Digest, 30 (2), 91-105, (1998). https://dx.doi.org/10.1177/058310249803000201

7 Sohn, H., Farrar, C. R., Hemez, F. M., Shunk, D. D., Stinemates, D. W., Nadler, B. R. and Czarnecki, J. J. Technical Report LA-13976-MS, Los Alamos National Laboratory, USA, A review of structural health monitoring literature: 1996-2001, (2003).

8 Thatoi, D. N., Das, H. C. and Parhi, D. R. Review of techniques for fault diagnosis in damaged structure and engi- neering system, Advances in Mechanical Engineering, 4, 327569, (2012). https://dx.doi.org/10.1155/2012/327569

9 Worden, K., Manson, G. and Fieller, N. R. J. Damage detection using outlier analysis, Journal of Sound and Vibration, 229 (3), 647-667, (2000). https://dx.doi.org/10.1006/jsvi.1999.2514

10 Hsu, T.-Y. and Loh, C.-H. Damage detection accommodating nonlinear environmental effects by nonlinear principal component analysis, Structural Control and Health Monitoring, 17 (3), 338-354, (2010). https://dx.doi.org/10.1002/stc.320

11 Figueiredo, E., Park, G., Farrar, C. R., Worden, K. and Figueiras, J. Machine learning algorithms for damage detection under operational and environmental variability, Structural Health Monitoring, 10 (6), 559-572, (2011). https://dx.doi.org/10.1177/1475921710388971

12 Kim, C. W., Kitauchi, S., Chang, K. C., McGetrick, P. J., Sugiura, K. and Kawatani, M., (2014), Structural damage diagnosis of steel truss bridges by outlier detection. Safety, Reliability, Risk and Life-Cycle Performance of Structures and Infrastructures, chap. 620, pp. 4631-4638, CRC Press. https://dx.doi.org/10.1201/b16387-670

13 Santos, A., Figueiredo, E., Silva, M. F. M., Sales, C. S. and Costa, J. C. W. A. Machine learning algorithms for damage detection: Kernel-based approaches, Journal of Sound and Vibration, 363, 584-599, (2016). https://dx.doi.org/10.1016/j.jsv.2015.11.008 
14 Meruane, V. and Heylen, W. Structural damage assessment under varying temperature conditions, Structural Health Monitoring, 11 (3), 345-357, (2012). https://dx.doi.org/10.1177/1475921711419995

15 Shu, J., Zhang, Z., Gonzalez, I. and Karoumi, R. The application of a damage detection method using artificial neural network and train-induced vibrations on a simplified railway bridge model, Engineering Structures, 52, 408-421, (2013). https://dx.doi.org/10.1016/j.engstruct.2013.02.031

16 Yarnold, M. and Moon, F. Temperature-based structural health monitoring baseline for long-span bridges, Engineering Structures, 86, 157-167, (2015). https://dx.doi.org/10.1016/j.engstruct.2014.12.042

$17 \mathrm{Li}, \mathrm{J}$. and Hao, H. Health monitoring of joint conditions in steel truss bridges with relative displacement sensors, Measurement, 88, 360-371, (2016). https://dx.doi.org/10.1016/j.measurement.2015.12.009

18 Zhang, Q. and Zhou, Y. Investigation of the applicability of current bridge health monitoring technology, Structure and Infrastructure Engineering, 3 (2), 159-168, (2007). https://dx.doi.org/10.1080/15732470600590762

19 Cavadas, F., Smith, I. F. and Figueiras, J. Damage detection using data-driven methods applied to moving-load responses, Mechanical Systems and Signal Processing, 39 (1), 409-425, (2013). https://dx.doi.org/10.1016/j.ymssp.2013.02.019

20 Lanata, F. and Del Grosso, A. Damage detection and localization for continuous static monitoring of structures using a proper orthogonal decomposition of signals, Smart Materials and Structures, 15 (6), 1811, (2006). https://dx.doi.org/10.1088/0964-1726/15/6/036

21 Kerschen, G. and Golinval, J.-C. Physical interpretation of the proper orthogonal modes using the singular value decomposition, Journal of Sound and Vibration, 249 (5), 849865, (2002). https://dx.doi.org/10.1006/jsvi.2001.3930

22 Rao, A. R. M., Lakshmi, K. and Venkatachalam, D. Damage diagnostic technique for structural health monitoring using POD and self adaptive differential evolution algorithm, Computers \& Structures, 106, 228-244, (2012). https://dx.doi.org/10.1016/j.compstruc.2012.05.009

23 Shane, C. and Jha, R. Proper orthogonal decomposition based algorithm for detecting damage location and severity in composite beams, Mechanical Systems and Signal Processing, 25 (3), 1062-1072, (2011). https://dx.doi.org/10.1016/j.ymssp.2010.08.015

24 Brunell, G. and Kim, Y. J. Effect of local damage on the behavior of a laboratory-scale steel truss bridge, Engineering Structures, 48, 281-291, (2013). https://dx.doi.org/10.1016/j.engstruct.2012.09.017
25 Kim, Y. J. and Yoon, D. Identifying critical sources of bridge deterioration in cold regions through the constructed bridges in north dakota, Journal of Bridge Engineering, 15 (5), 542-552, (2009). https://dx.doi.org/10.1061/(asce)be.1943-5592.0000087

${ }^{26}$ Leskovec, J., Rajaraman, A. and Ullman, J. D., Mining of massive datasets, Cambridge University Press (2014). https://dx.doi.org/10.1017/CBO9781139924801.012

27 Farrar, C. R., Sohn, H. and Worden, K. Data normalization: A key for structural health monitoring, in Proceedings of the Third International Structural Health Monitoring Workshop, pp. 1229-1238, Stanford, California, DEStech Publications, Inc., Lancaster, Palo Alto., (2001). http://permalink.lanl.gov/object/tr?what=info:lanlrepo/lareport/LA-UR-01-4212

28 Madenci, E. and Guven, I., The finite element method and applications in engineering using ANSYS $\mathrm{R}$, Springer (2015). https://dx.doi.org/10.1007/978-1-4899-7550-8

29 Arya, A. S. and Ajmani, J. L., Design of steel structures, Nem Chand (2001).

30 Bridge rules, (2008). http://www.sr.indianrailways.gov.in/ uploads/files/1332924439677-bridge_rule.pdf

31 Wang, H., Tao, T., Cheng, H. and He, X. Simulation study on train-induced vibration control of a long-span steel truss girder bridge by tuned mass dampers, Mathematical Problems in Engineering, (2014). https://dx.doi.org/10.1155/2014/506578

32 Poh, K. Stress-strain-temperature relationship for structural steel, Journal of Materials in Civil Engineering, 13 (5), 371-379, (2001). https://dx.doi.org/10.1061/(asce)08991561(2001)13:5(371)

33 Sampaio, R., Maia, N., Almeida, R. and Urgueira, A. A simple damage detection indicator using operational deflection shapes, Mechanical Systems and Signal Processing, 72, 629-641, (2016). https://dx.doi.org/10.1016/j.ymssp.2015.10.023

34 Sohn, H., Czarnecki, J. A. and Farrar, C. R. Structural health monitoring using statistical process control, Journal of Structural Engineering, 126 (11), 1356-1363, (2000). https://dx.doi.org/10.1061/(asce)07339445(2000)126:11(1356)

35 Yan, A.-M., Kerschen, G., De Boe, P. and Golinval, J.C. Structural damage diagnosis under varying environmental conditions-Part I: A linear analysis, Mechanical Systems and Signal Processing, 19 (4), 847-864, (2005). https://dx.doi.org/10.1016/j.ymssp.2004.12.002 